\title{
Obstacle Avoidance Using CTC and Virtual Structure for Leader-Follower Formation on Mobile Robot
}

\author{
Maskhur Zulkarnain \\ Department of Electrical Engineering \\ Institut Teknologi Sepuluh Nopember \\ Surabaya, Indonesia \\ maskhurzulkarnain@gmail.com
}

\author{
Trihastuti Agustinah \\ Department of Electrical Engineering \\ Institut Teknologi Sepuluh Nopember \\ Surabaya, Indonesia \\ trihastuti@ee.its.ac.id
}

\begin{abstract}
This paper examined the development of the combination of virtual structure and leader-follower as an obstacle avoidance method in the formation control of mobile robots. The robot formation is designed with the Separation Bearing Control approach between the leader robot and the virtual robot. The virtual robot is used as a virtual follower and a reference trajectory for the follower robot. When the follower robot detects an obstacle, the follower robot trajectory is adjusted using a trajectory planner for obstacle avoidance. After passing through the obstacle, the follower robot will track its position back in formation using virtual robot position and heading as reference. The leader and the follower robots are perturbed by disturbances. In order to ensure the achievement of small tracking error, a controller is designed using the integration of kinematic and dynamic controllers with a disturbance observer. The kinematic and dynamic controllers are designed using input-output linearization method and computed by torque control. Based on the simulation results, it is obtained that the MSE value for all tests, both without obstacles, with obstacles, and with disturbances is always smaller than $1 \times 10^{-4} \mathrm{~m}$. Meanwhile, with the use of the disturbance observer, the results of the kinematic and dynamic errors are almost the same as the tracking and obstacle avoidance tests without disturbance, with a MSE difference of $0.001 \mathrm{~m}$. The effectiveness of the proposed method is verified by the simulation results.
\end{abstract}

Keywords-CTC, leader follower, obstacle avoidance, SBC, virtual structure.

\section{INTRODUCTION}

The robot formation has been developed along with the development of technology and the need for coordination systems of multiple robots particularly in the military field. The formation of robots also plays an important role for the transportation of an object, field inspection, and supervision of an area. The formation controls have been developed in terms of both strategy formation and stability control formation. There are three basic methods of formation strategy, which are behaviour, leader-follower, and virtual structure approach [1]. Leader-follower formation approach can be applied based on the relative movement of each state of the mobile robot to form the formation during tracking a reference or maneuver position [2]. In order to maintain the formed formation, SBC and SSC approach can be used [3]. The follower robot position and heading are designed with respect to the leader robot using $l-\varphi$ and $l-l$ control strategy. SBC is used to maintain and adjust the distance and separation bearing angle of follower and SSC is used to maintain a distance between robots. This method is also effective to avoid singularity conditions, which can occur when the robots in formation collide or the distance becomes infinite.

The collision can also occur when there is a disturbance in the robot kinematics or dynamics. There are several approaches to solve this problem. In [4], to reduce the effect of disturbance on robot kinematics, the controller is designed using a backstepping method and disturbance observer. This disturbance observer can attenuate disturbance regardless of the tracking error results. The disturbance observer can also be used for robot dynamics [5, 6 , 7]. By using a disturbance observer, the value of disturbance can be estimated and used to compensate for the effect of disturbance. In [8], a computed torque control and nonlinear disturbance observer are used for the follower robot in formation while performing trajectory tracking. However, the leader robot can also be affected by disturbances. Another proposed method to solve the disturbance problem is using a virtual structure based on a linear algebra approach [9]. Virtual structure is designed with a spring-damper approach to maintain formation and a linear algebraic approach to coordinate the robots in formation. However, with the control variable is each robot velocity and the parameter virtual is designed using gain for its error velocities, when robot dynamics is disturbed by disturbance, the reference velocities generated from the kinematic controller will also be disturbed. Thus, the disturbance will still affect the robots and their formation.

In addition to the internal collisions, there are also external factors which are obstacles [10]. In most environments there are obstacles that can interfere with robot formation $[11,12]$. Thus, an avoidance approach is needed. Obstacle avoidance problems in robot formation are discussed in [13]. In this study, the $l-\varphi$ control strategy was used to regulate the formations and the artificial potential field method was used to avoid obstacles. Then in [14], a combination of leader-follower strategy and virtual structure are used to manage and maintain formation when there are obstacles in the robot trajectory. This combination 
method can also be used to accelerate the convergence rate of a system [15].

Based on the mentioned problems, this study proposes the development of the combination of virtual structure and leader-follower approach for trajectory tracking. The virtual robot is designed to form desired formation with leader robot using SBC approach. Then the trajectory of virtual robot will be used as reference to the kinematic controller of follower robots. When there is an obstacle in trajectory, the follower will avoid it and then will track its position back in formation. Since the virtual robot is not affected by disturbance and obstacles, the formation can be maintained if the tracking error of the leader rand follower robot is small. To ensure small tracking error is achieved, the controller is designed based on input-output linearization method and computed torque control. The computed torque control is designed with positive gain constant and PID. With the presence of disturbances, the disturbance observer is designed to estimate its value in order to reduce the effect on the robot.

\section{METHODS}

This section will explain the design of each block diagram including the kinematic and dynamic controllers, formation control approach, virtual structure, and obstacle avoidance methods.

The combination of virtual structure and leaderfollower approach is shown in Fig. 1, 2, and 3 which are leader robots $(\mathrm{RL})$, follower robots $\left(\mathrm{RF}_{1}, \mathrm{RF}_{2}\right)$, and virtual robots $\left(\mathrm{RV}_{1}, \mathrm{RV}_{2}\right)$.

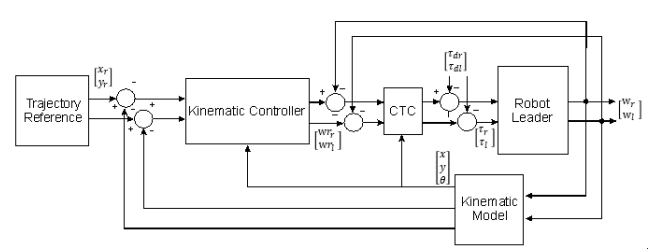

Fig. 1. Control of the leader robot.

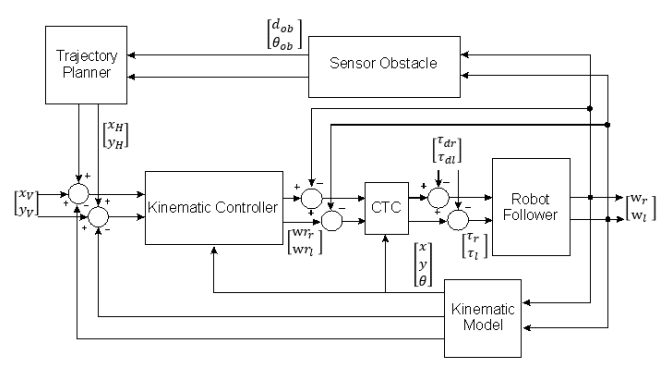

Fig. 2. Control of the follower robot.

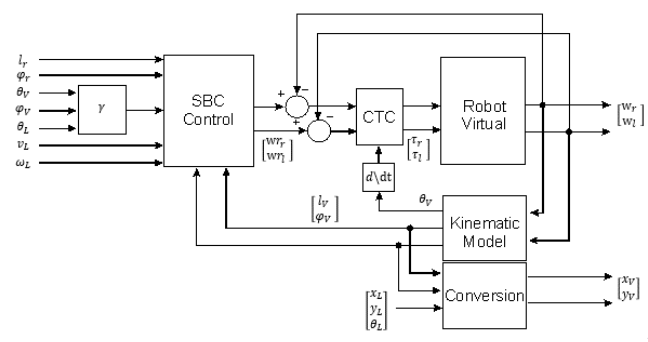

Fig. 3. Control of the virtual robot.

\section{A. Dynamics Controller}

The dynamics model of RL and RF are given as follows:

$$
\bar{M}(q) \dot{\eta}+\bar{V}(q, \dot{q}) \eta=\bar{B}(q) \tau_{F}+\tau_{d}
$$

Where $\tau_{d}$ is disturbance. Model for RV is using (1) by ignoring the disturbance factor.

The dynamic controller is designed using computed torque control (CTC) as follows.

$$
\begin{gathered}
\tau_{L}=\bar{B}^{-1}\left(\bar{M}(q)\left[K_{r} \dot{\eta}+K_{p} e+K_{i} \int e d t+K_{d} \frac{d e}{d t}\right]+\right. \\
\bar{V}(q, \dot{q}) \eta)
\end{gathered}
$$

To compensate the disturbance in (1), the disturbance estimation, $\tau_{e s}$, is added to (2) as follows:

$$
\begin{gathered}
\tau_{F}=\bar{B}^{-1}\left(\bar{M}(q)\left[K_{r} \dot{\eta}+K_{p} e+K_{i} \int e d t+K_{d} \frac{d e}{d t}\right]+\right. \\
\left.\bar{V}(q, \dot{q}) \eta-\tau_{e s}\right)
\end{gathered}
$$

Where the variables are as follows:

$$
\begin{array}{ll}
\tau_{e s} & =\alpha+\beta \\
\dot{\alpha} & =L\left(\bar{V}(q, \dot{q}) \eta-\bar{B}(q) \tau-\bar{\tau}_{d}\right) \\
\beta & =C * \eta \\
L(q) & =C \bar{M}(q)^{-1}
\end{array}
$$

Where $L(q)$ is the observer gain.

\section{B. Formation control and Robot Virtual Kinematic Controller Design}

The kinematic controller is designed based on SBC approach for formation control between RL-RV and inputoutput linearization (IOL) for each robot in formation. The SBC approach, which is an $l-\varphi$ control, will be used as a method to adjust each RV with respect to the RL as shown in Fig. 4.

The equations of $l$ and $\varphi$ can be expressed as:

$$
\begin{aligned}
l= & \sqrt{\left(x_{L}-x_{V}-d \cos \theta_{V}\right)^{2}+\left(y_{L}-y_{V}\right)^{2}} \\
\varphi= & \pi-\operatorname{atan} 2\left(y_{V}+d \sin \theta_{V}-y_{L}, x_{L}-x_{V}-\right. \\
& \left.d \cos \theta_{V}\right)-\theta_{L}
\end{aligned}
$$

The derivative of (5) and (6) can be written as:

$$
\begin{aligned}
& \dot{l}=v_{V} \cos \gamma-v_{V} \cos \varphi+\omega_{V_{12}} d \sin \gamma \\
& \dot{\varphi}=\frac{v_{L} \sin \varphi}{l}-\frac{v_{L} \sin \gamma}{l}+\frac{\omega_{V} d \cos \gamma}{l}-\omega_{L}
\end{aligned}
$$

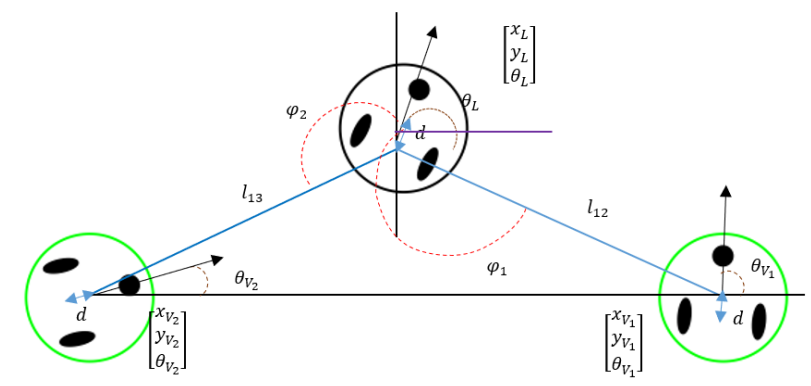

Fig. 4. SBC approach. 
With

$\gamma=\theta_{L}-\theta_{F}+\varphi$

Where $\omega_{L}=\dot{\theta}_{L}$ and $\omega_{F}=\dot{\theta}_{F}$.

Using IOL, the control input of $\mathrm{RV}_{1}$ and $\mathrm{RV}_{2}$ can be obtained as shown in (10).

$$
\begin{aligned}
{\left[\begin{array}{c}
v_{V} \\
\omega_{V}
\end{array}\right]=} & {\left[\begin{array}{cc}
\cos \gamma & d \sin \gamma \\
-\frac{\sin \gamma}{l} & \frac{d \cos \gamma}{l}
\end{array}\right]^{-1}\left(\left[\begin{array}{c}
k_{1}\left(l^{r}-l\right) \\
k_{2}\left(\varphi^{r}-\varphi\right)
\end{array}\right]-\right.} \\
& {\left.\left[\begin{array}{cc}
-\cos \varphi & 0 \\
\frac{\sin \varphi}{l} & -1
\end{array}\right]\left[\begin{array}{l}
v_{L} \\
\omega_{L}
\end{array}\right]\right) }
\end{aligned}
$$

Where $k_{1}$ and $k_{2}$ are constant gain.

\section{Leader and Follower Kinematic Controller Design}

The kinematic controller is used to generate robot velocity which can be written as follows:

$$
\begin{aligned}
{\left[\begin{array}{c}
v_{L} \\
\omega_{L}
\end{array}\right]=} & {\left[\begin{array}{cc}
\cos \theta_{L} & -d \sin \theta_{L} \\
\sin \theta_{L} & d \cos \theta_{L}
\end{array}\right]^{-1} } \\
& {\left[\begin{array}{l}
\dot{x}_{r}+k_{L}\left(x_{r}-x_{L}\right) \\
\dot{y}_{r}+k_{L}\left(y_{r}-y_{L}\right)
\end{array}\right] } \\
{\left[\begin{array}{c}
v_{F} \\
\omega_{F}
\end{array}\right]=} & {\left[\begin{array}{cc}
\cos \theta & -d \sin \theta \\
\sin \theta & d \cos \theta
\end{array}\right]^{-1} } \\
& {\left[\begin{array}{l}
\dot{x}_{V}+\dot{x}_{H}+k_{F}\left(x_{V}+x_{H}-x_{F}\right) \\
\dot{y}_{V}+\dot{y}_{H}+k_{F}\left(y_{V}+y_{H}-y_{F}\right)
\end{array}\right] }
\end{aligned}
$$

Where $d$ is the distance between the center point of the robot and the midpoint between the right and left wheels, $k_{L}$ and $k_{F}$ are gain constants, $\left(x_{r}, y_{r}\right)$ are the reference trajectory, and $\left(x_{V}, y_{V}\right)$ are the RV trajectory. The value of $x_{H}$ and $y_{H}$ will be determined for obstacle avoidance.

\section{Obstacle Avoidance Design}

The shape of the obstacle and the sensor measurement area used in this paper is designed as shown in Fig. 5, where the distance of the RF to the obstacle can be calculated when the RF is at a certain radius. When the robot is at the radius measurement, the sensor will measure the distance and angle of the robot with respect to obstacles as shown in Fig. 6.

The following equation is used to calculate the value of heading robot with respect to obstacle:

$$
\theta_{o b}=\operatorname{atan} 2\left(y_{f}-y_{o b}, x_{f}-x_{o b}\right)
$$

The value of heading measurement will be used to determine the direction of movement robot for avoidance. Then the value of $d_{o b}$ is used to determine the change in the trajectory of the follower robot $(\mathrm{RF})$.

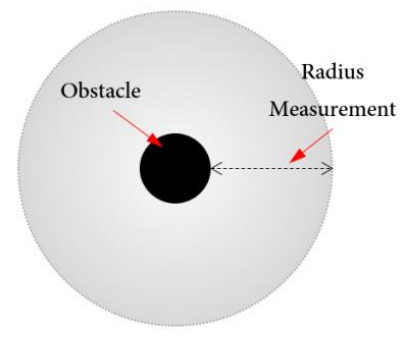

Fig. 5. Obstacle design and distance measurement.

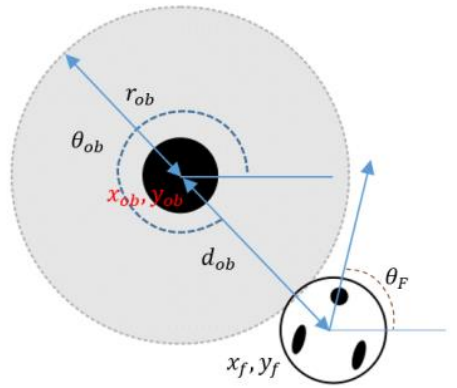

Fig. 6. The distance and robot heading with respect to obstacles.

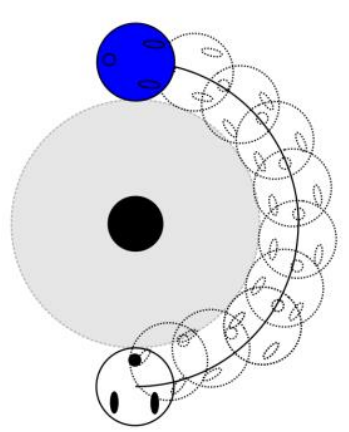

(a)

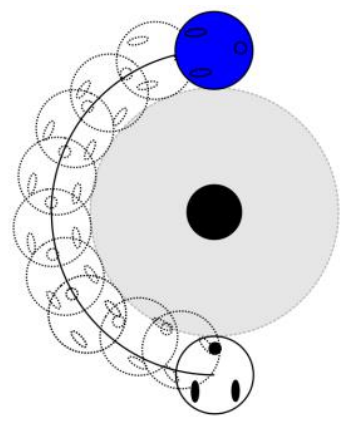

(b)
Fig. 7. Illustration of a semicircle trajectory: (a) $R F_{1}$ and (b) $R F_{2}$

\section{E. Tracking with Semicircle Trajectory}

The design of the first trajectory change is a semicircle trajectory. The new trajectory coordinates of the RF are given in (14).

$$
\begin{aligned}
& x_{F}=x_{F}+\left(r_{o b} \cos \left(\theta_{o b} \pm \theta_{s t l}\right)\right. \\
& y_{F}=x_{F}+\left(r_{o b} \sin \left(\theta_{o b} \pm \theta_{s t l}\right)\right.
\end{aligned}
$$

Where $\theta_{s t l}=10^{\circ}, 20^{\circ}, \ldots, 90^{\circ}$. The difference between $\mathrm{RF}_{1}$ and $\mathrm{RF}_{2}$ is the polarity value of $\theta_{\text {stl }}$. For more details can be seen in Fig. 7.

As it is shown in Fig. 7, $\mathrm{RF}_{1}$ will move to the right and $\mathrm{RF}_{2}$ will move to the left every time its sensor detects an obstacle. After the RF is in the end position, the reference will change to the trajectory of RV. There will be no collision between followers assuming the tracking error is small or close to zero.

\section{F. Tracking with Half Square Trajectory}

The design of the next trajectory for obstacle avoidance is designed with a half square trajectory. The coordinates of the trajectory are determined by the angle measurement value, $\theta_{o b}$, and the heading robot, $\theta_{F}$, for example $2 \pi-$ $\theta_{o b}-\theta_{F}>0^{\circ}$ and $80^{\circ} \leq \theta_{F} \leq 90^{\circ}$.

\section{SimULATION AND RESULT}

\section{A. Simulation Model using Matlab}

In this paper, Simulink and Matlab 2020b 64-bit are used for implementing the design in section II. There are 3 mobile robots and 2 virtual robots used, which are $R L, R_{1}$, $\mathrm{RF}_{2}, \mathrm{RV}_{1}$, and $\mathrm{RV}_{2}$. The virtual robots are used as a reference for each follower robot. The robot's kinematics and dynamics are controlled by the kinematic and dynamic 
controller. For RV, there will be a conversion block from $l$ and $\varphi$ parameter to $x y$ robot in cartesian coordinate using $\mathrm{RL}$ as reference.

The robot parameters used are $r=0.025 \mathrm{~m}, b=$ $0.1 \mathrm{~m}, d=0.05 \mathrm{~m}, m_{c}=3 \mathrm{~kg}, m_{w}=0.01 \mathrm{~kg}, I_{c}=$ $1.5625 \mathrm{kgm}^{2} \quad, \quad I_{w}=0.0005 \mathrm{kgm}^{2} \quad, \quad$ and $I_{m}=$ $0.00025 \mathrm{kgm}^{2}$. For the SBC formation parameter, the reference value used for $\mathrm{RV}_{1}$ is $l_{12}=0.7745$ and $\varphi_{1}=$ $3.926 \mathrm{rad}$ then for $\mathrm{RV}_{2}$ is $l_{13}=0.7745$ and $\varphi_{2}=$ $2.356 \mathrm{rad}$.

\section{B. Tracking on 8-shaped Reference}

This test was carried out without any disturbance and the plants used in (1) and (2), and for kinematic controllers of RL, RV, and RF used in (10) - (12). The gain parameters used in the experiment were obtained by trial and error. The gain parameters on each robot are the kinematic controller gain and dynamic controller gain including $K_{r}, K_{p}, K_{d}$, and $K_{i}$.

For the first test, the chosen gain parameters are $K_{L}=120, K_{p L}=70, K_{d L}=0.48, K_{i L}=800$, and $K_{r L}=0.14$ and the step size sample time used in Simulink is $0.01 \mathrm{~s}$. In this test, the speed of the right and left wheels is limited to \pm 100 $\mathrm{rad} / \mathrm{s}$ or $2.5 \mathrm{~m} / \mathrm{s}$ with $r=0.025 \mathrm{~m}$.

The kinematic error of RL values obtained by both $e_{x}$ and $e_{y}$ are good with the maximum error for $e_{x}$ and $e_{y}$ is $1.7 \times 10^{-4} \mathrm{~m}$ around $t=0.02 \mathrm{~s}$ (see Fig. 8). Although there is oscillation, the value is small, about $\pm 0.5 \times 10^{-4} \mathrm{~m}$. The MSE values obtained from $e_{x}$ and $e_{y}$ are very small, around $9.29 \times 10^{-10}$ and $4.18 \times 10^{-10}$.

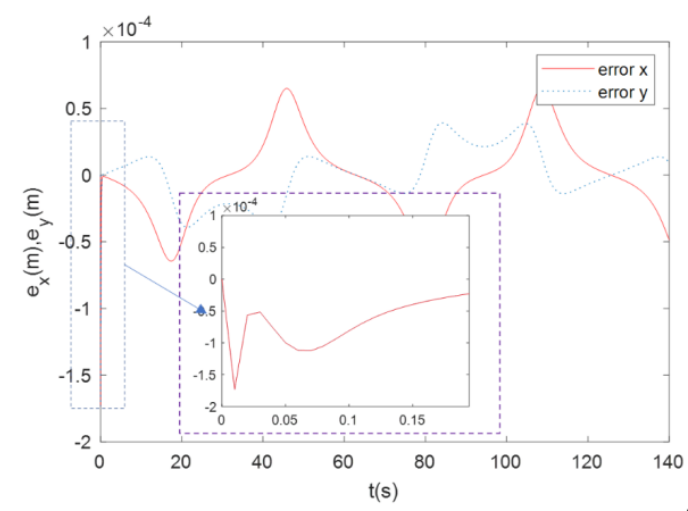

Fig. 8. Kinematic error of RL(8-shaped tracking).

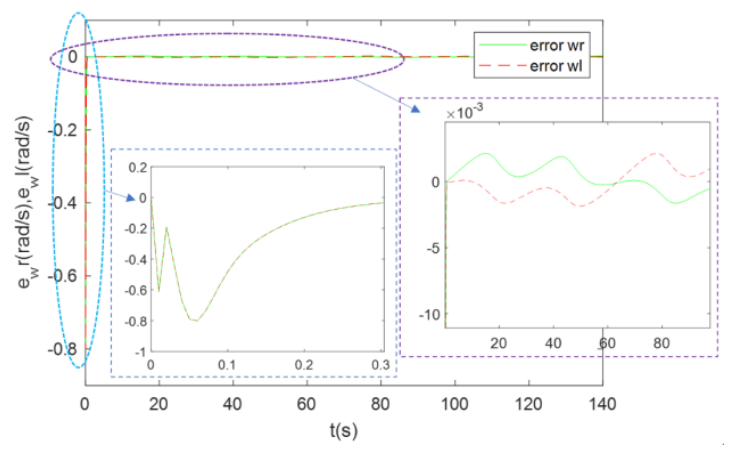

Fig. 9. Dynamics error of RL(8-shaped tracking).
In general the error obtained is small, therefore it is assumed that the obtained maximum error is still within the tolerance limit and the desired steady-state error is achieved.

In Fig. 9, it is shown that the right wheel speed error of RL, $e_{w r}$, and the left wheel speed error, $e_{w l}$, in $\mathrm{rad} / \mathrm{s}$ unit. The MSE results are about $3.2793 \times 10^{-4}$ and $3.2792 \times$ $10^{-4}$. The error results oscillations also occur but are still within the tolerance limit which is $\pm 2 \times 10^{-3}$ at steady-state condition.

\section{Tracking of Robot Follower}

In this test, the gain parameters for $R L, R_{1}$, and $R V_{2}$ are used the same as the previous test, while the gain parameters for $\mathrm{RF}_{1}$ and $\mathrm{RF}_{2}$ are set the same except for $K_{r F 2}$ is 0.4 . In this test, the results to be analyzed are data from $\mathrm{RF}_{1}$ and $\mathrm{RF}_{2}$.

The kinematic and dynamics error of $\mathrm{RF}_{1}$ are shown in Fig. 10 and 11. In the kinematic error, the MSE is $8.8935 \times 10^{-9}$ and $6.2992 \times 10^{-9}$ for $e F 1_{x}$ and $e F 2_{y}$. The MSE values are greater than RL's result because there is an overshoot and undershoot on the reference signal as explained before but the peak error of the kinematic error $\mathrm{RF}_{1}$ is smaller than RL about $-6.7 \times 10^{-3} \mathrm{~m}$. The settling time value of $e F 1_{y}$ is also smaller than $e_{y}$ which is $0.1204 \mathrm{~s}$ while the settling time of $e F 1_{x}$ is greater than $e_{x}$.

In the dynamics error, settling time and MSE values of $\mathrm{RF}_{1}$ on average are greater than RL's result for both $e_{w r}$ and $e_{w l}$. The overall error obtained from this test is quite good.

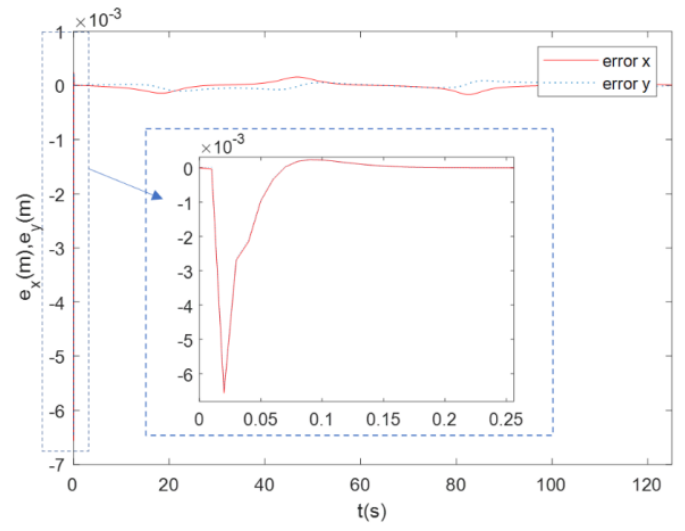

Fig. 10. Kinematic error of $\mathrm{RF}_{1}$ (8-shaped tracking).

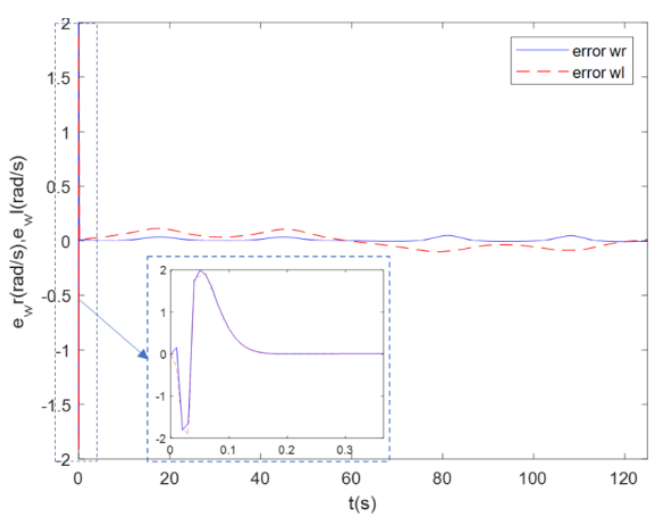

Fig. 11. Dynamics error of $\mathrm{RF}_{1}$ (8-shaped tracking). 


\section{Circle Trajectory Test}

This test is carried out without disturbance with the same parameters and criteria used are the same as the previous test. The reference trajectories used are $x=4 \sin (t / 20)$ and $y=4 \sin (t / 20)$ and initial error $\theta_{V 1}$ and $\theta_{V 2}$ are $45^{\circ}$.

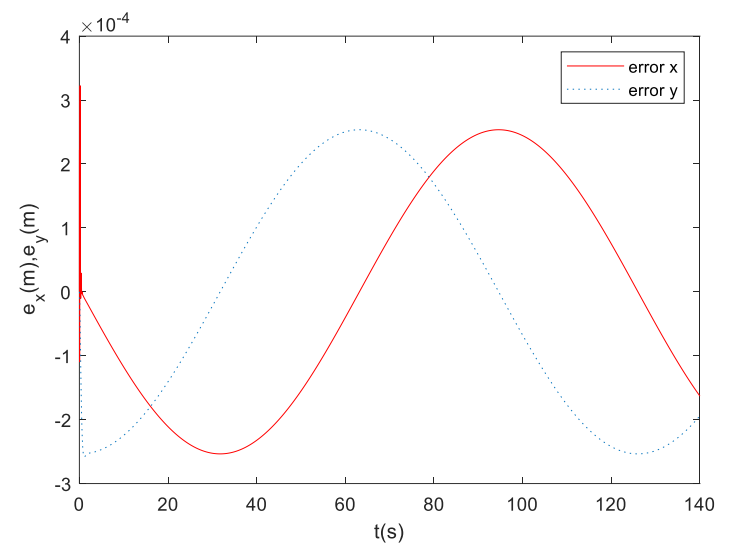

Fig. 12. Kinematic error of RL (circle trajectory).

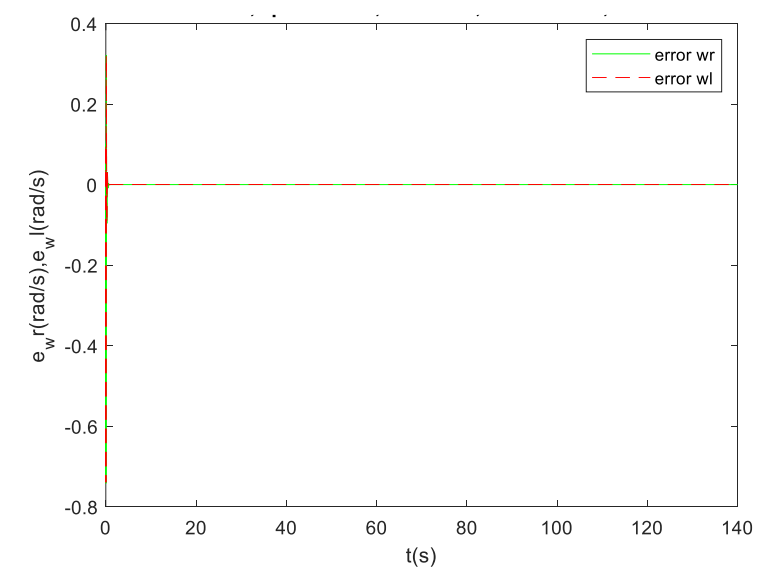

Fig. 13. Dynamic error of RL (circle trajectory).

\section{E. Tracking Robot Leader Trajectory Testing (Circle Trajectory)}

The gain parameters used in the fourth test are $K_{L}=10$, $K_{p L}=30, K_{d L}=0.47, K_{i L}=1100$, dan $K_{r L}=0.7$ and the step sample time value used is $0.01 \mathrm{~s}$. The results of the test are shown in Fig. 12 and 13.

Fig. 12 shows that the kinematic error results obtained are quite good with a peak error around $\pm 3.1 \times 10^{-4}$. The MSE value obtained is small, about $2.9868 \times 10^{-8}$ and $3.4276 \times 10^{-8}$. With a peak error less than $\pm 0.8 \mathrm{rad} / \mathrm{s}$ as shown in Fig. 13, the speeds of the right and left wheels are within the desired tolerance of $\pm 100 \mathrm{rad} / \mathrm{s}$ or $2.5 \mathrm{~m} / \mathrm{s}$. The value of settling time is $0.3947 \mathrm{~s}$ and $0.3938 \mathrm{~s}$ while the MSE values of $e_{w r}$ and $e_{w l}$ is $2.5 \times 10^{-3}$ and $4.8 \times 10^{-3}$.

\section{F. Robot Follower Tracking Trajectory on Circle Trajectory}

The results of the test tracking trajectory using the gain parameters $K_{F 1}=60, K_{p F 1}=80, K_{d F 1}=1.1 K_{i F 1}=100$, and $K_{r F 1}=0.4$. In $e F 1_{y}$, the data obtained is still not good, but the peak error value is still within the desired limit of $2.35 \times 10^{-3}$ as well as the dynamic error results as shown in Fig. 15, the values of $e F 1_{w r}$ and $e F 1_{w l}$ are quite good.
As for the kinematic error on $\mathrm{RF}_{2}$ Fig. 16, the value is quite good with the MSE values for $e F 2_{x}$ and $e F 2_{y}$ respectively $4.4108 \times 10^{-8}$ and $4.1978 \times 10^{-8}$ then MSE for error the dynamics are $8 \times 10^{-3}$ and $8.1 \times 10^{-3}$. From this test, it can be seen that even though the initial error is given at the angle, the speed of the right and left wheels does not exceed the given limit of $100 \mathrm{rad} / \mathrm{s}$.

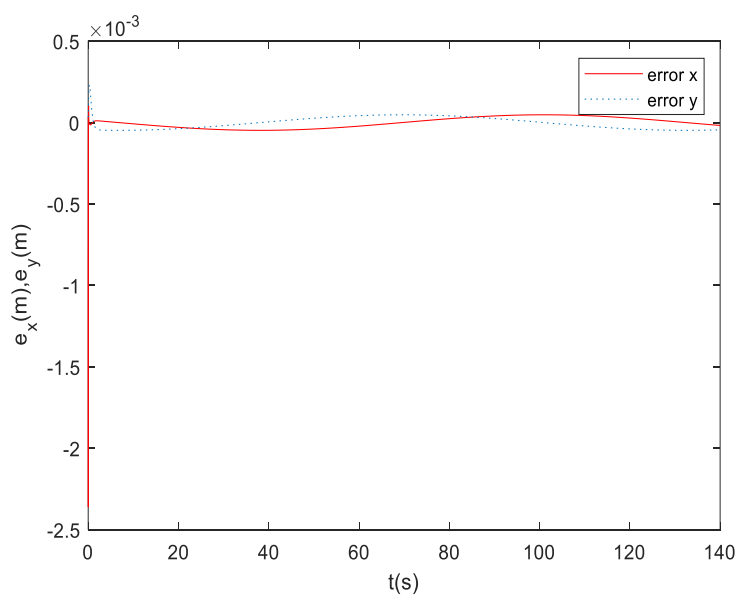

Fig. 14. Kinematic error of $\mathrm{RF}_{1}$ (circle trajectory).

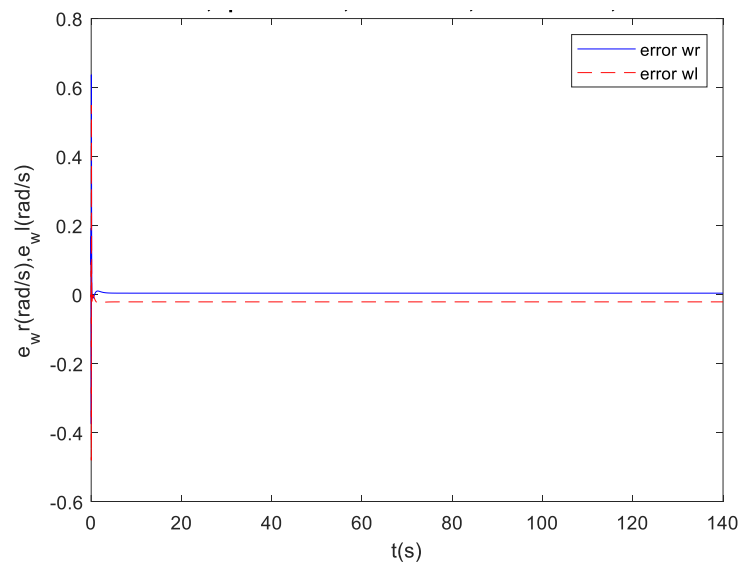

Fig. 15. Dynamics error of $\mathrm{RF}_{1}$ (circle trajectory).

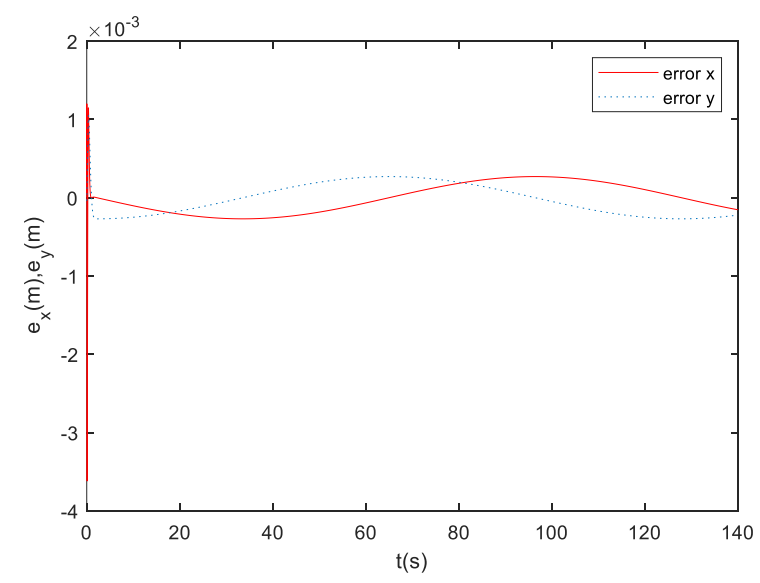

Fig. 16. Kinematic error of $\mathrm{RF}_{2}$ (circle trajectory). 


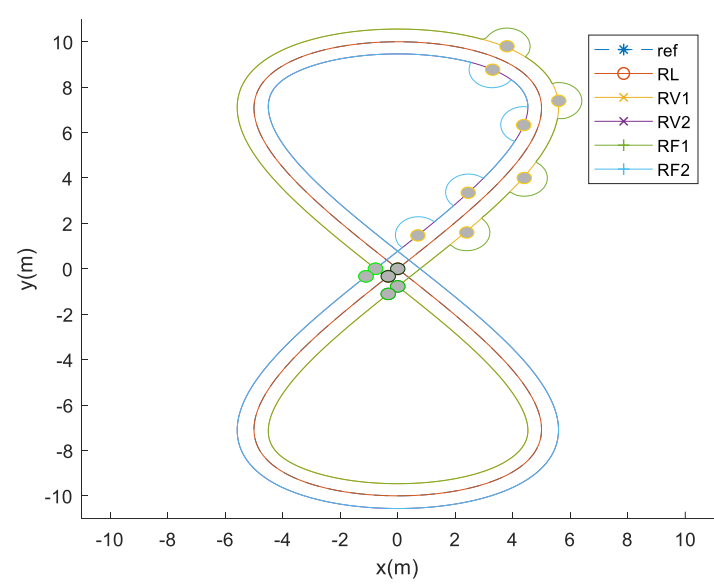

Fig. 17. Tracking trajectory test results with obstacles.

\section{G. Tracking Trajectory with Obstacle Avoidance}

There are 8 obstacles in this test, the speed value of the right and left wheels is limited to $\pm 100 \mathrm{rad} / \mathrm{s}$. In this test, the initial position error given to the RV and follower is quite small, which is $0.25 \mathrm{~m}$ for each robot.

The $\mathrm{RF}_{1}$ and $\mathrm{RF}_{2}$ managed to avoid the obstacles given to the path. The kinematic and dynamics errors of $\mathrm{RF}_{1}$ are given in Figs. 18-19. Whenever the robot detects an obstacle, which is set to $0.8 \mathrm{~m}$, the trajectory of $\mathrm{RF}_{1}$ will change into a semicircle. Because the angle of the robot can be different each time it detects an obstacle, the trajectory must be adjusted to avoid collisions between robots. The kinematic error and dynamics of $\mathrm{RF}_{2}$ are shown in Figs. 2021. The kinematic error of $\mathrm{RF}_{2}$ is smaller than those of $\mathrm{RF}_{1}$. However, the dynamic error exceeds $100 \mathrm{rad} / \mathrm{s}$ even though the right and left wheel speeds have been limited to \pm 100 $\mathrm{rad} / \mathrm{s}$.

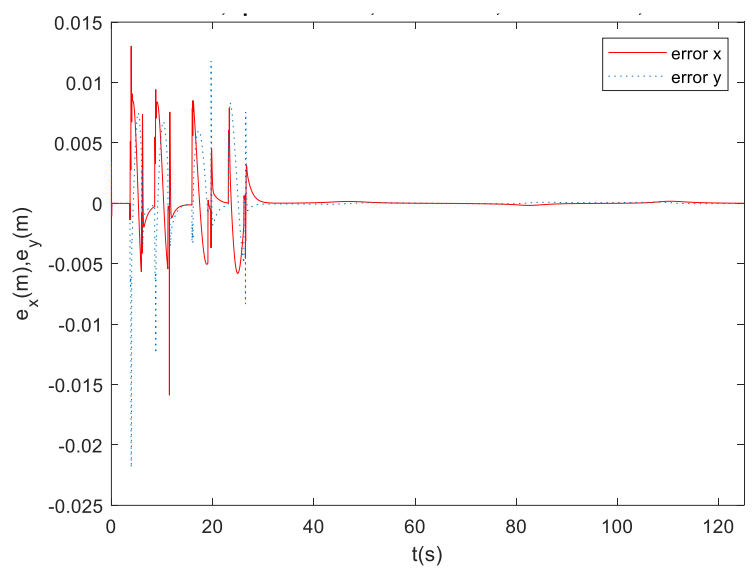

Fig. 18. Kinematic error of $\mathrm{RF}_{1}$ with obstacle avoidance.

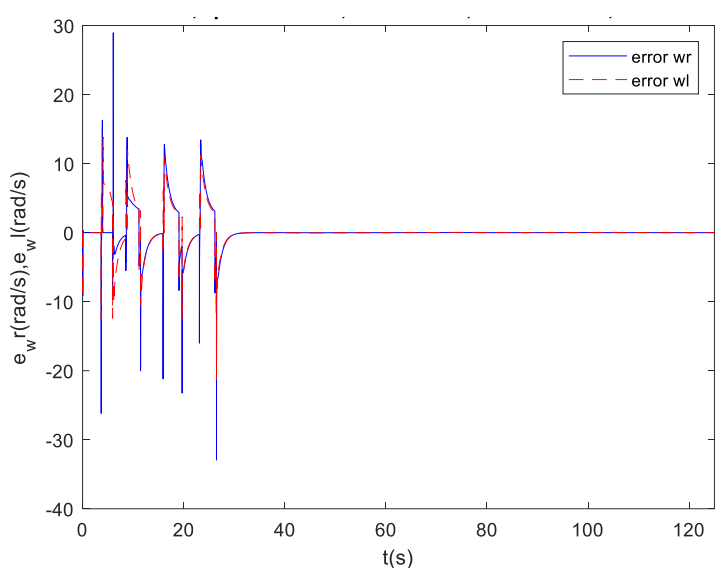

Fig. 19. Dynamics error of $\mathrm{RF}_{1}$ with obstacle avoidance.

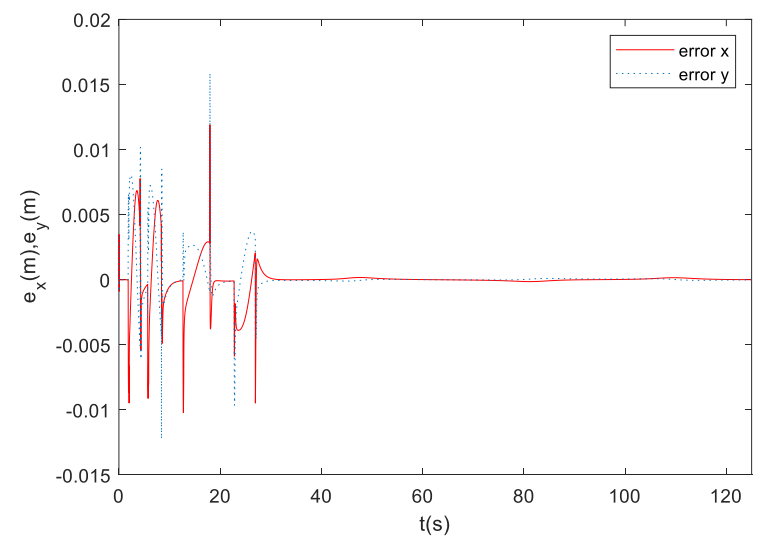

Fig. 20. Kinematics Error $\mathrm{RF}_{2}$ with obstacle avoidance.

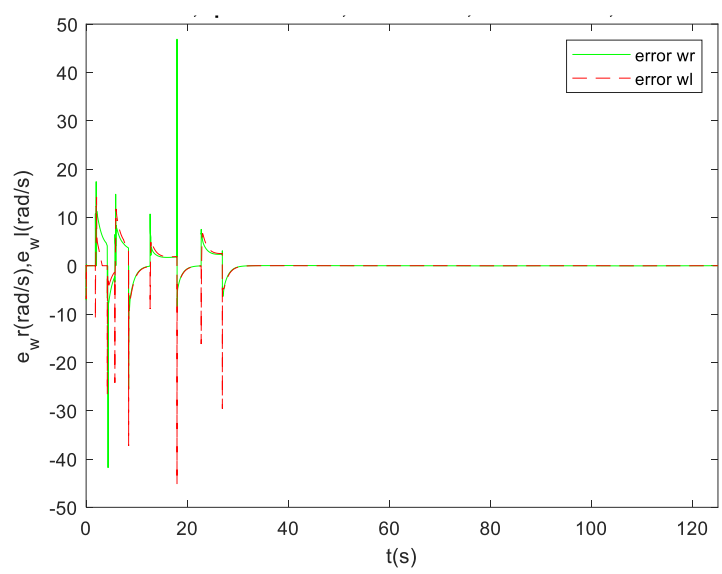

Fig. 21. Dynamics error of $\mathrm{RF}_{2}$ with obstacle avoidance.

The result of the tracking with obstacles and disturbance observers is shown in Fig. 22. In which the follower robot (RF) can avoid the given obstacle. The kinematic error and dynamic error of $\mathrm{RF}_{1}$ are shown in Figs. 23-24. Although every time a robot tracks a new reference for obstacle avoidance there is a rapid change in error, the error reduces to a smaller error. The speed limit for this tracking is limited to $35 \mathrm{rad} / \mathrm{s}$ but the results show that the given speed limit does not exceed the limit in the dynamic error. 


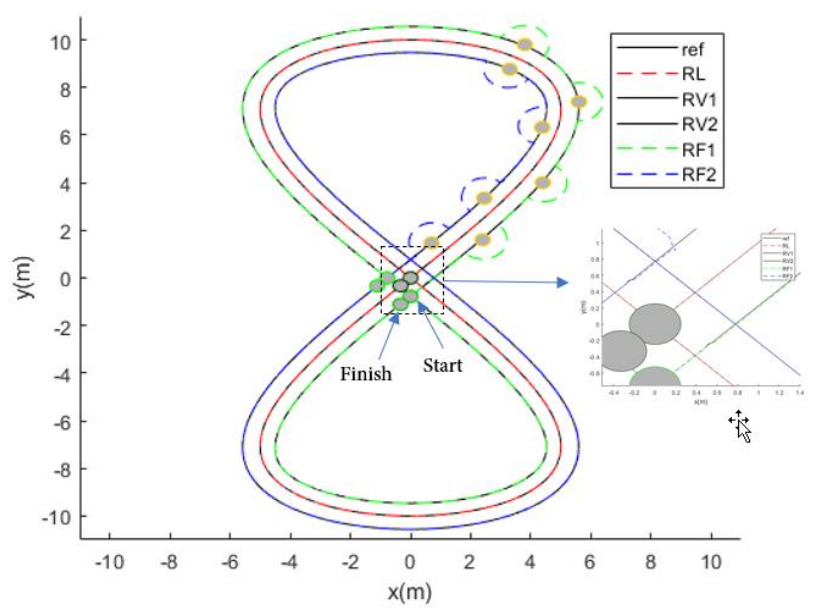

Fig. 22. Tracking with obstacles and disturbance observers.

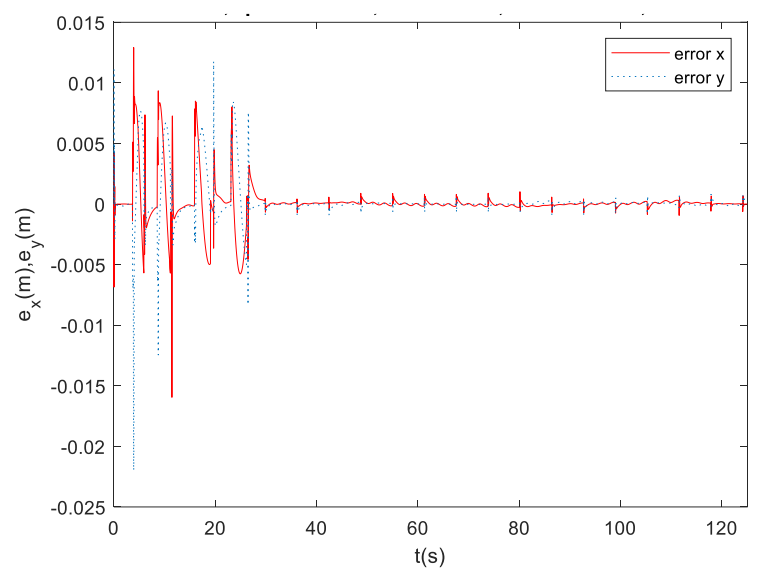

Fig. 23. Kinematic error of $\mathrm{RF}_{1}$ (tracking with obstacles and disturbance observers).

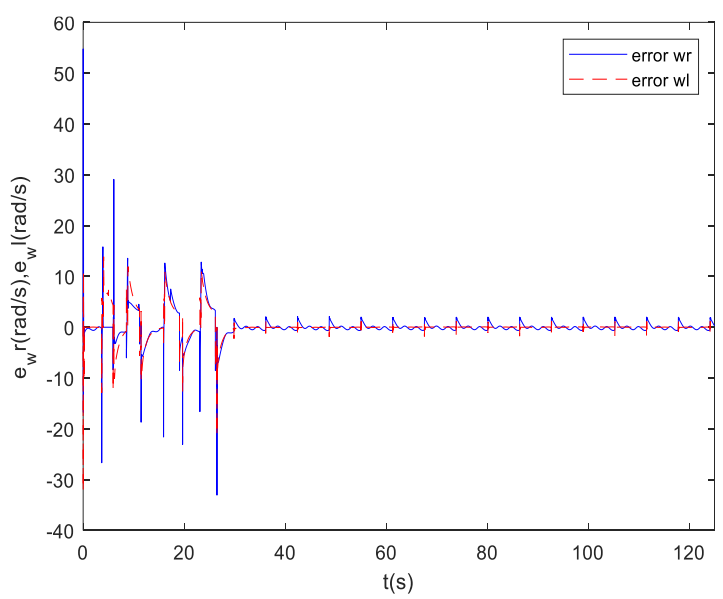

Fig. 24. Dynamics error of $\mathrm{RF}_{1}$ (tracking with obstacles and disturbance using disturbance observers).

In this test, the difference can be seen when a disturbance observer is used to reduce the effect of the disturbance, although the errors in each tracking reference for obstacle avoidance stay the same but overall performance of the controller is good based on the achieved desired target.

\section{CONCLUSION}

The simulation results show that the proposed method can be used to solve obstacle avoidance problems on trajectory tracking of formation control. When there are no obstacles, the formation can be formed very fast and is maintained with small kinematic and dynamic errors. Both in a circle and an 8-shaped trajectory, good tracking performance is achieved. In the presence of obstacles, the kinematic and dynamic error values change rapidly every time the follower robots avoid the obstacle, particularly at the start of the trajectory change but within the desired value. For the disturbance system problem, with a disturbance observer, the result of tracking the trajectory in both trajectories is very good. From the test results, the MSE value for all tests, both with obstacles, without obstacles, and with disturbances is always smaller than $1 \times 10^{-4} \mathrm{~m}$. Meanwhile, with the use of the disturbance observer, the results of the kinematic and dynamic errors are almost the same as the tracking and obstacle avoidance tests without disturbances with a MSE difference of $0.001 \mathrm{~m}$. Thus, the development of the formation approach and controller can be used for trajectory tracking with obstacle avoidance and disturbance problems in formation control.

\section{REFERENCES}

[1] B. A. Issa and A. T. Rashid, "A Survey of Multi-mobile Robot Formation Control," International Journal of Computer Applications, vol. 181, no. 48, p. 0975 - 8887, 2019.

[2] M. Ghiasvand and K. Alipour, "Formation control of wheeled mobile robots based on fuzzy logic and system dynamics," Fuzzy Systems (IFSC), 2013 13th Iranian Conference, p. 1-6, 2013.

[3] S. Ahmed, M. N. Karsiti and R. N. K. Loh, "Control Analysis and Feedback Techniques for Multi Agent Robots," INTECH Open Access Publisher, 2019.

[4] M. A. M. Obaid, A. R. Husain and A. A. M. Al-kubati, "Robust Backstepping Tracking Control of Mobile Robot Based on Nonlinear Disturbance Observer," International Journal of Electrical and Computer Engineering (IJECE)2, vol. 6, no. 2, pp. 901-908, 2016.

[5] D. Huang and J. Zhai, "Trajectory Tracking Control of Wheeled Mobile Robots Based on Disturbance Observer," Chinese Automation Congress (CAC), pp. 1761-1765, 2015.

[6] M. Noorbad, A. M. Shahri, K. Shojaei and B. Tabibian, “A Passivity based Stability Analysis of Disturbance Observer based Trajectory Tracking Control of Nonholonomic Wheeled Mobile Robot," IEEE/ASME International Conference on Advanced Intelligent Mechatronics, pp. 477-482, 2010.

[7] Y. Jinhua and Y. Suzhen, "Trajectory Tracking control of WMR Based on Sliding Mode Disturbance Observer with Unknown Skidding and Slipping," International Conference on Cybernetics, Robotics and Control, pp. 18-22, 2017.

[8] A. Kusumawardana and T. Agustinah, "Disturbance Compensation Using CTC with NDOB for Formation Control Mobile Robots," International Conference on Information and Communications Technology, 2018.

[9] Arfittariah and T. Agustinah, "Formation Control of Multi-Robot using Virtual Structures with a Linear Algebra Approach," JAREE (Journal on Advanced Research in Electrical Engineering), 2020.

[10] T. Miyazaki and K. Takaba, "Formation control of mobile robots with obstacle avoidance," International Conference on Control, Automation and Systems (ICCAS), pp. 121-126, 2014.

[11] S. Xiao, L. Feng, H. Lian and B. Du, "Dynamic Formation and Obstacle Avoidance Control for Multi Robot System," World Congress on Intelligent Control and Automation (WCICA), pp. 5963, 2016.

[12] H. Melujerdi, M. Saad, and J. Ghommam, "Multi mobile robots formation in presence of obstacles," IEEE International Conference on Mechatronics, pp. 510-515, 2011. 
[13] Z. Ying and L. Xu, "Leader-follower Formation Control and Obstacle Avoidance of Multi-robot Based on Artificial Potential Field," IEEE Chinese Control and Decision Conference (CCDC), 2015.

[14] A. Abbaspour, S. A. A. Moosavian and K. Alipour, "A Virtual Structure-based Approach to Formation Control of Cooperative Wheeled Mobile Robots," RSI/ISM International Conference on Robotics and Mechatronics, 2013.

[15] L. Dong, Y. Chen and X. Qu, "Formation Control Strategy for Nonholonomic Intelligent Vehicles Based on Virtual Structure and Consensus Approach," Procedia Engineering, p. 415 - 424. 